\title{
Grain-based products, food structure and health potential: holism vs reductionism
}

\section{Editorial}

Undeniably, technological treatments alter the physical structure of the food matrix and hence its nutritional value, either in a positive or negative way. There is obviously no question of eating cereal grains or legumes without hydrothermal treatment because starch has to be a minimally gelatinized to be digestible. Moreover, excessive refining followed by recombination of isolated ingredients generally leads to very energy-dense and nutritionally-poor quality foods, as in the case of our usual white wheat bread. Technological processes must be used wisely to maximize the nutritional value of plant products and not degrade it. ${ }^{1}$

The reductionist approach used in research in food science and human nutrition explains this fact. The food was indeed reduced to a single sum of compounds leading to study each compound alone and to summarize the health potential of foods to only some compounds (e.g. milk and calcium, meat and protein, citrus fruits and vitamin $\mathrm{C}$, etc.). The role of the food matrix in the nutritional effect of plant foods has been largely neglected leading to food ingredient fractionationrecombination processes and/or excessive refining. Instead, a holistic approach to food considers the food matrix as a complex structure that plays a role on the health effects through the satiety potential, the different release kinetics of nutrients and possible synergistic effects of the compounds in the human body. According to this view, food is a complex set of macro, micro and phyto-nutrients in permanent complex interaction: in other words, a holistic vision of the food is: $1+1>2$ and not $1+1=2 .{ }^{2}$ Therefore, in grains and seeds, beyond the single chemical composition, physical structure of the food matrix plays an important role in their nutritional and health effects. ${ }^{3}$

The nutritional quality of food products of plant origin can be modified by numerous more or less drastic technological processes. Among the drastic methods are especially distinguished extrusion cooking and refining. These two treatments generally reduce the nutritional value of plant products by either drastically reducing the levels of bioactive compounds of interest (nutrient density) via refining or by deconstructing the original food matrix and gelling starch excessively (extrusion-cooking at high temperatures and pressures). Today, less drastic technological treatments (i.e. minimal processing) are sought to preserve both the physical structure of plant products (effect on satiety) and nutrient density in bioactive compounds such as fiber, vitamins, minerals and phytonutrients.

Moreover, knowing the functional properties of certain ingredients, we now know how to make more slowly digestible starch (carbohydrates) to reduce the glycemic response. Among the technological treatments reported less drastic or preserving a certain naturalness of the food, the use of pre-hydrolysis of the fibers allows rendering them more soluble and viscous to act on glycemic and lipemic responses. Pre- fermentation and/or germination of food is also widely used, particularly in developing countries, to increase the nutrient density in bioactive compounds by releasing their bound fraction in the food matrix or generating-via bacterial metabolism novel compounds of interest. ${ }^{1,4}$

Today, with relatively simple technology (that could also be
Volume I Issue I - 2014

\author{
Anthony Fardet \\ Department of Human Nutrition, National Institute of \\ Agronomic Research (INRA), France
}

\begin{abstract}
Correspondence: Anthony Fardet, Department of Human Nutrition, National Institute of Agronomic Research (INRA), BP 10448, F-63000 Clermont-Ferrand, Clermont University, University of Auvergne, UMR I019, UNH, CRNH Auvergne, France, Tel +33(0)473299687,

Email anthony.fardet@clermont.inra.fr
\end{abstract}

Received: April 12, 2014 | Published: April 23, 2014

called soft as opposed to conventional and drastic hydrothermal treatments at high pressures), it is known how to increase the levels of carbohydrates and bioactive compounds of certain foods, including cereal and legume seeds. These modifications are based on a reduction of the enzymatic availability of the starch, the activation of endogenous enzymes in the food and/or the use of exogenous hydrolytic enzymes. ${ }^{1}$ From the standpoint of preventive nutrition, these treatments allow developing food reducing hyperglycemia, reducing hyperlipidemia, increasing mineral bioavailability, increasing concentrations of bioavailable antioxidants or reducing the allergenicity potential of certain proteins. ${ }^{1}$ Moreover, these treatments allow a relative preservation of the initial structure of the physical plant food matrix as opposed to fractionation, refining and then recombining the separate ingredients. However, a functional food alone cannot solve everything and prevent all metabolic dysregulations associated with overeating: they must register under balanced diets promoting dietary diversity and consumption of less refined foods combining lower energy density and higher nutrient density (in bioavailable bioactive compounds).

Such a "shift" in the transformation of plant products can only be done by developing a more holistic vision of the food that more respects its natural complexity and therefore its long-term health potential. Indeed, food compounds are not drugs and human preventive nutrition is not pharmacology!

\section{Acknowledgements}

None.

\section{Conflict of interest}

Author declares that there is no conflict of interest.

\section{References}

1. Fardet A, Souchon I, Dupont D. Structure des aliments et effets nutritionnels. France: Quae Publisher. 2013.

2. Fardet A, Rock E. The search for a new paradigm to study micronutrient and phytochemical bioavailability: from reductionism to holism. Med Hypotheses. 2014;82(2):181-186. 
3. Fardet A. New hypotheses for the health-protective mechanisms of wholegrain cereals: what is beyond fibre? Nutr Res Rev. 2010;23(1):65-134.
4. Poutanen K, Flander L, Katina K. Sourdough and cereal fermentation in a nutritional perspective. Food Microbiol. 2009;26(7):693-699. 